\title{
Characterization of single-domain antibodies against Foot and Mouth Disease Virus (FMDV) serotype $O$ from a camelid and imaging of FMDV in baby hamster kidney- 21 cells with single-domain antibody-quantum dots probes
}

\author{
Di Wang, Shunli Yang, Shuanghui Yin", Youjun Shang, Ping Du, Jianhong Guo, Jijun He, Jianping Cai \\ and Xiangtao Liu ${ }^{*}$
}

\begin{abstract}
Background: Foot-and-mouth disease (FMD) is a highly contagious disease that affects cloven-hoofed animals and causes significant economic losses to husbandry worldwide. The variable domain of heavy-chain antibodies (VHHs or single domain antibodies, sdAbs) are single-domain antigen-binding fragments derived from camelid heavy-chain antibodies.

Results: In this work, two sdAbs against FMD virus (FMDV) serotype $O$ were selected from a camelid phage display immune library and expressed in Escherichia coli. The serotype specificity and affinity of the sdAbs were identified through enzyme-linked immunosorbent assay and surface plasmon resonance assay. Moreover, the sdAbs were conjugated with quantum dots to constitute probes for imaging FMD virions. Results demonstrated that the two sdAbs were specific for serotype $O$ and shared no cross-reactivity with serotypes $A$ and Asia 1. The equilibrium dissociation constant (KD) values of the two sdAbs ranged from $6.23 \mathrm{nM}$ to $8.24 \mathrm{nM}$, which indicated high affinity to FMDV antigens. Co-localization with the sdAb-AF488 and sdAb-QD probes indicated the same location of FMDV virions in baby hamster kidney-21 (BHK-21) cells.
\end{abstract}

Conclusions: sdAb-QD probes are powerful tools to detect and image FMDV in BHK-21 cells.

Keywords: Foot-and-mouth disease virus serotype O, Immune library, Single domain antibody, Quantum dots, Probe, Imaging

\section{Background}

Foot-and-mouth disease (FMD), a highly contagious disease from the FMD virus (FMDV), causes significant economic losses on husbandry worldwide. In particular, the 1997 FMD outbreak in Taiwan brought a loss of approximately US\$ 1.6 billion to the swine industry [1]. In addition, the 2001 FMDV outbreak in Great Britain caused tremendous losses to the agricultural and tourism industries [2]. Thus, FMD is classified as

\footnotetext{
*Correspondence: yinshuanghui@caas.cn; liuxiangtao@caas.cn State Key Laboratory of Veterinary Etiological Biology, National Foot and Mouth Diseases Reference Laboratory, Lanzhou Veterinary Research Institute, Chinese Academy of Agricultural Sciences, Lanzhou, Gansu, China
}

an International Epizootic Office (OIE) list A disease and is under strict control. FMD is also known as the first animal disease in China. FMDV belongs to the family Picornaviridae and mainly infects cloven-hoofed animals. The genome of this virus is a single-stranded RNA (approximately $8.5 \mathrm{~kb}$ ) that is surrounded by a protein coat consisting of four structure proteins, namely, VP1, VP2, VP3, and VP4 [3]. FMDV is divided into seven immunologically distinct serotypes: A, O, C, Asia I, and South African Territories (SAT1, SAT 2, and SAT3) [4]. These seven serotypes share no cross-immunity. That is, animals that have previously been infected with one serotype remain susceptible to the six other serotypes. 
Occurring in Europe, South America, and Asia, FMDV serotype $\mathrm{O}$ is the most globally prevalent of the seven serotypes.

Immunoglobulins in mammals are commonly assembled from two identical light (L) chains and two identical heavy $(\mathrm{H})$ chains. The $\mathrm{N}$-terminal domains of the $\mathrm{H}$ and $\mathrm{L}$ polypeptide chains are responsible for antigen recognition. Apart from these conventional antibodies, naturally occurring and functional antibodies that are devoid of light chains are also found in the sera of camelids [5] and a cartilaginous fish [6]. These special antibodies, also known as heavy chain antibodies (HCAbs), lack the first constant domain. The variable domain of the heavy chain from HCAbs (sdAbs or single-domain antibodies) is fully qualified to bind antigens like the Fab fragment (antigen binding fragment) of conventional antibodies. Given their small size, high physicochemical stability, facile genetic manipulation, and capability to bind epitopes inaccessible to conventional antibodies, sdAbs are ideal antigen-binding elements [7]. In addition, sdAbs are highly expressed and easily purified in Escherichia coli [8], yeasts [9], mammalian cell lines, and plants [10]. Thus, these antibodies have various medical and biotechnological applications, such as in cancer therapy $[11,12]$.

Fluorescent semi-conductor nanocrystals, also known as quantum dots (QDs), are composed of atoms from elements belonging to groups II to VI (e.g., CdSe, CdTe, $\mathrm{CdS}$, and $\mathrm{ZnSe}$ ) or III to $\mathrm{V}$ (e.g., InP and InAs) in the periodic table. The electronic characteristics of QDs are closely related to its size and shape [13]. Compared with the classical organic dyes, QDs have broader excitation spectra, narrower emission spectra, brighter and more stable fluorescence, and longer lifetimes; thus, QDs can facilitate the long-term bio-imaging of living tissues and cells [14-16]. QD-conjugated antibodies have been used as probes to detect the interaction between viruses and target cells and for the cellular imaging of cancer cells $[17,18]$. In the present study, we panned and characterized sdAbs against FMDV serotype $\mathrm{O}$ antigens from an immune camelid phage display library. The general construction procedures of a sdAb phage library and the panning procedures of antigen-specific sdAbs are shown in Fig. 1. Results showed that the special sdAbs exhibited a high affinity to FMDV serotype $\mathrm{O}$ and shared no cross-reactivity with serotypes A and Asia 1 . We also investigated the possibility of constructing sdAb-QD probes for imaging FMDV in BHK-21 cells.

\section{Methods}

Camelus bactrianus immunization and antibody detection Two C. bactrianus were vaccinated with $2 \mathrm{~mL}$ of inactivated FMDV serotype $O$ vaccine $\left(10^{7} 50 \% \mathrm{TCID}_{50} / \mathrm{mL}\right)$ emulsified in Montanide ISA 206 (ISA) in accordance with previously described methods [18]. The concentration used was based on the weight ratio between porcine and bactrianus camels. Booster immunization was conducted on days $21,28,35$, and 42 . Serum samples were collected after each immunization to monitor the humoral immune response by using a liquid-phase blocking enzyme-linked immunosorbent assay (ELISA). All the FMDV strains, standard sera, and ELISA kit in this work were provided by the OIE/CHINA National Foot and Mouth Disease Reference Laboratory.

All animals were handled in strict accordance with the Animal Ethics Procedures and Guidelines of the People's Republic of China, and this study was approved by the Animal Ethics Committee of Lanzhou Veterinary Research Institute, Chinese Academy of Agricultural Sciences (No. LVRIAEC2012-006). In this study, the owner of the camels allowed the use of the animals in the experiments. Considerable effort was exerted to minimize suffering.

\section{Separation of peripheral mononuclear lymphocyte cells}

Ficoll-Paque gradient centrifugation is a conventional method to isolate peripheral blood lymphocyte cells (PBLCs) [19]. Seven days after the final inoculation, $100 \mathrm{~mL}$ of peripheral blood was collected from the external jugular vein in ethylene diamine tetraacetic acid (EDTA)-coated tubes $(50 \mathrm{~mL}$ from each camel). The blood samples were mixed with an equal volume of Hank's buffer and then added into centrifuge tubes that contained appropriate amounts of lymphocyte separation medium. After centrifugation (2000 rpm, $20 \mathrm{~min}$ ) using a horizontal centrifuge, the target layer was isolated, and the cells in this layer were suspended in minimal essential medium. The cells were incubated at $37^{\circ} \mathrm{C}$ in six-well plates to eliminate cell debris. Subsequently, the purified monoclonal cells were divided into aliquots of $6 \times 10^{8}$ PBLCs and then stored in liquid nitrogen.

\section{SdAb library construction}

Total RNA was extracted using RNeasy Plus Mini Kit (Qiagen), and first-strand cDNA was synthesized with oligo-dT primers (Takara). The sdAb fragments were amplified in accordance with previously described procedures [18]. The target fragments that encode HCAbs were amplified with primers LH21 and LH22 (Table 1), which were annealed at both the leader sequences and the $\mathrm{CH} 2$ sequences. The fragments $(600 \mathrm{bp})$ from the first amplification were used as templates for the next amplification with primers VHU and VHD, which were annealed at the framework 1 and 4 regions of $\mathrm{VHH}$. The primers VHHSfiI and VHHNotI, which contain the restriction enzyme cutting sites, were used in the amplification for future steps. The products were digested with restriction enzymes, cloned into the phage vector 


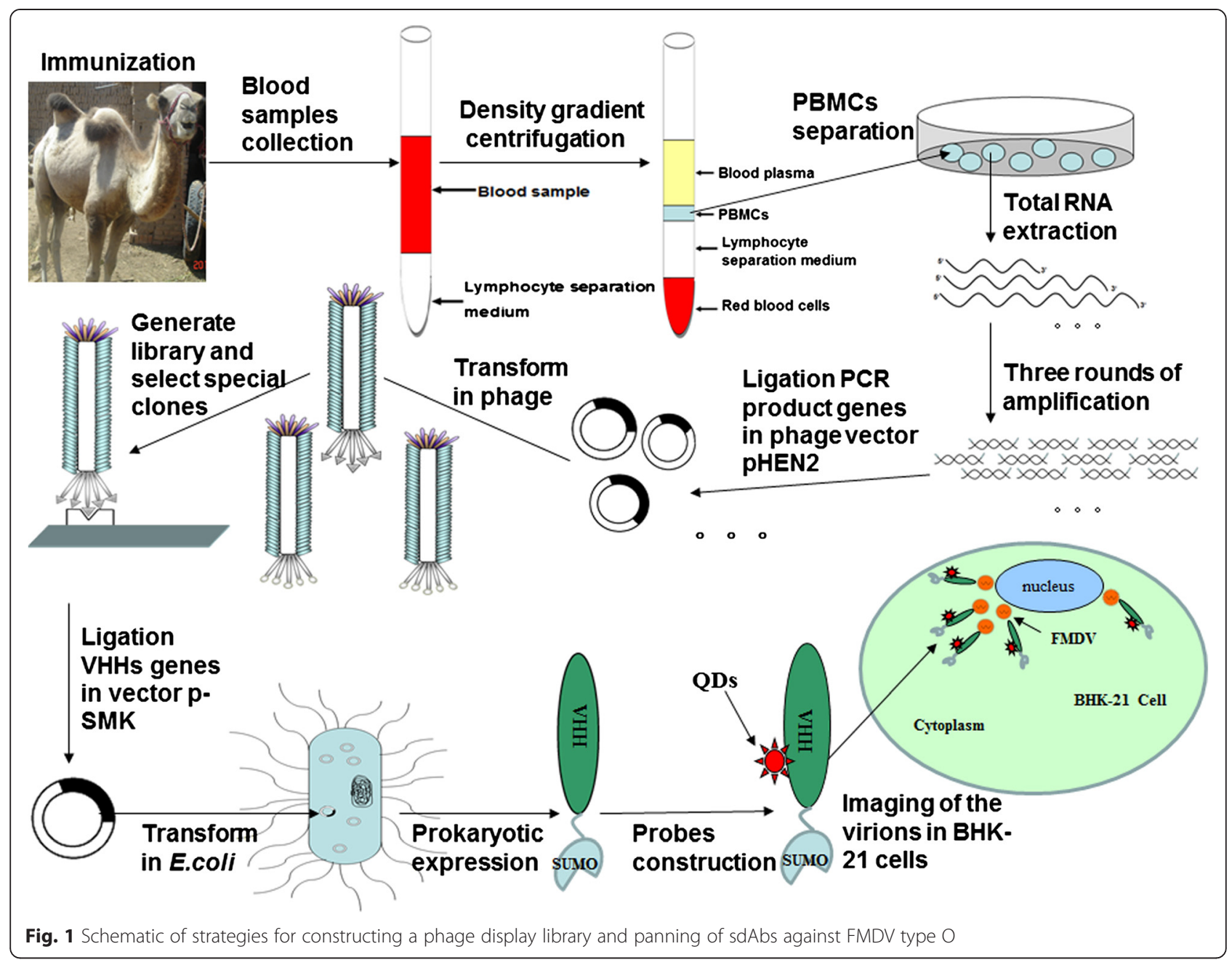

pHEN2 [20-22] with T4 DNA ligase (New England Biolabs, UK), and then transformed into E. coli TG1 cells. The size and diversity of the sdAb library were estimated by plating on LB-ampicillin $(100 \mu \mathrm{g} / \mathrm{mL})$ agar plates, followed by colony PCR with a universal primer pair. The colonies from the plated cells were cultured, subpackaged, and then stored at $-80{ }^{\circ} \mathrm{C}$ in $\mathrm{LB}$ medium supplemented with $50 \%$ glycerine for further use.

Panning of special sdAbs against FMDV type 0

In accordance with the principle of phage display [23], the library was infected with M13K07 helper phage and

Table 1 Primers in this work

\begin{tabular}{|c|c|c|}
\hline Primer & Primer sequences $\left(5^{\prime}-3^{\prime}\right)$ & Restriction site \\
\hline $\mathrm{LH} 21$ & GTCCTGGCTGCTCTTCTACAAGG & None \\
\hline LH22 & GGTACGTGCTGTTGAACTGTTCC & None \\
\hline VHU & GCCATGGCTSAKGTBCAGCTGSTGGAGTCTGG & None \\
\hline VHD & GCGGCTGAGGAGACGGTGACCWGG & None \\
\hline VHHSfil & ATTACTGGCCCAGCCGGCCGGCTGAKGTBCAGCTGGTGGAGTCTGGRGGAGGCT & Sfil \\
\hline VHHNotl & ATTATTAGTGCGGCCGCTGAGGAGACGGTGACCWGGGTCC & Notl \\
\hline D01U & CTTGGTCTCTGATGTGCAGCTGGTGGAGTC & Bsal \\
\hline D01D & CTCGGATCCTCATGAGGAGACAGTGACCTGG & BamHI \\
\hline A01U & CTTGGTCTCTGATGTGCAGCTGGTGGAGTC & Bsal \\
\hline A01D & CTCGGATCCTCATGAGGAGACGGTGACCTGG & $\mathrm{BamHI}$ \\
\hline
\end{tabular}


the phage particles were rescued and precipitated with polyethylene glycol. The virus-like particle antigen of FMDV type $\mathrm{O}$ was coated overnight at $4{ }^{\circ} \mathrm{C}$ at a concentration of $1 \mathrm{mg} / \mathrm{mL}$ in carbonate-buffered saline to pan the phages that bind to the antigen-coated immunotubes. The library was subjected to four rounds of panning. To select the $\mathrm{VHH}$ antibodies with high affinity, four rounds of panning were performed by increasing the washing time and the concentration of Tween-20 in PBS. The concentrations of Tween- 20 were $0.1 \%, 0.2 \%$, $0.3 \%$, and $0.4 \%$ in the first, second, third, and fourth rounds, respectively. The enriching factors of each panning round were calculated on the basis of the input and output phages. Individual clones that were randomly selected from the four rounds of panning were subjected to monoclonal phage ELISA [24] for detecting the affinity to the target antigen. The plasmids of positive clones were extracted using a plasmid isolation kit (Qiagen Miniprep kit). Local DNA sequencing of unique clones was completed by Sangon Biotech (Shanghai) Co., Ltd.

\section{Expression and purification of recombinant sdAbs}

A p-SMK vector that was fused with a small ubiquitinlike modifier (SUMO) protein tag was employed to promote the expression, solubility, and correct folding of the protein [25]. The presence of a highly stable protein (SUMO) at the N-terminus of a partner protein increases the recombinant fusion protein yield [26]. The $\mathrm{sdAb}$ fragments were amplified with primers A01U, A01D, D01U, and D01D, and then cloned into p-SMK vectors after double digestion with restriction enzymes BsaI and BamHI. The recombinant plasmids were transformed into a BL21-codon-Plus (DE3)-RIL strain (Stratagene), and the cultures were incubated with a shaking culture at $37{ }^{\circ} \mathrm{C}$. The cultures were induced using isopropyl-b-thiogalactopyranoside when the $\mathrm{OD}_{600}$ ranged from 0.6 to 0.8 . After being incubated in a shaker incubator at $180 \mathrm{rpm}$ for $20 \mathrm{~h}$ at $20{ }^{\circ} \mathrm{C}$, the cells were collected through centrifugation and suspended in a $50 \mathrm{mM}$ Tris- $\mathrm{HCl}$ buffer ( $\mathrm{pH} 8.0)$. The recombinant bacteria were ultrasonically treated (three cycles, $10 \mathrm{~min} /$ cycle, working for $2 \mathrm{~s}$, and resting for $2 \mathrm{~s}$ ) to obtain the cell lysates. The proteins of interest were purified using NTA affinity resins (Qiagen) and then identified by SDS-PAGE.

Identification of recombinant sdAbs through Western blot After the separation of the protein mixture, Western blot was conducted using the Trans-Blot ${ }^{\circ}$ TurboTM Transfer System (Bio-Rad, USA). The polyvinylidene fluoride (PVDF) membrane that carried the protein mixture was washed thrice with PBST and blocked with $5 \%$ skim milk at $37^{\circ} \mathrm{C}$ for $1 \mathrm{~h}$. Horseradish peroxidase (HRP)conjugated anti-His monoclonal antibody (diluted 1:1000, Cwbiotech, China) was recognized as the primary antibody. The protein bands were detected with diaminobenzidine reagent.

Identification of serotype specificity using sandwich ELISA Sandwich ELISA was used to identify the serotype specificity of the sdAbs. The special sdAbs against FMDV type $\mathrm{O}$ were coated in the wells of 96-well microtiter plates to capture antibodies. The plates were incubated overnight at $4{ }^{\circ} \mathrm{C}$. Different viral antigens (inactivated FMDV type A, O, and Asia I, diluted to $16 \mu \mathrm{g} / \mathrm{mL}$ ) were added into the corresponding wells. After $1 \mathrm{~h}$ of incubation at $37{ }^{\circ} \mathrm{C}$, the wells were washed thrice with PBST. The corresponding serotype-specific rabbit antisera were subsequently added to all wells and incubated for another hour at $37{ }^{\circ} \mathrm{C}$. HRP-conjugated goat anti-rabbit IgG (1:1000) used as the secondary antibody was added into the wells after washing thrice with PBST. 3,3',5,5'Tetramethylbenzidine was used as the substrate for the colorimetric reaction, and the reaction was terminated with $2.0 \mathrm{M}$ sulfuric acid. The absorbance was measured at $450 \mathrm{~nm}$ with a microplate reader (Bio-Rad). The polyclonal antibodies against FMDV type $\mathrm{O}$ and the negative sera from FMDV-free camels were used as the positive and negative controls, respectively.

\section{Affinity of the sdAbs against FMDV type $O$}

Surface plasmon resonance (SPR) using Biacore 3000 (Biacore, Uppsala, Sweden) was utilized to identify the affinity to specific antigens. The carboxymethyl dextran surface of research-grade CM5 (GE healthcare) chips was activated with a $120 \mu \mathrm{L}$ mixture $(1: 1, \mathrm{v} / \mathrm{v})$ of $0.4 \mathrm{M}$ 1-ethyl-3-(3-dimethylaminopropyl) carbodiimide hydrochloride and $0.2 \mathrm{M}$ N-hydroxysuc-cinimide. Recombinant VP1 proteins were immobilized on the surface of reactive CM 5 sensor chips after being diluted to $25 \mu \mathrm{g} / \mathrm{mL}$ to obtain 3000 resonance units using the amine coupling kit. The sdAbs were diluted at concentrations of 25,50 , 75,100 , and $150 \mathrm{nM}$ with HBS-EP buffer and then added onto the sensor chips at a flow rate of $30 \mu \mathrm{L} / \mathrm{min}$. The binding kinetics were recorded, and the kinetic rate constants ( $\mathrm{ka}$ and $\mathrm{kd}$ ) were used to calculate the equilibrium dissociation constant (KD) [27]. Sensor chips were regenerated by $20 \mathrm{mM}$ glycinate hydrochloride $(\mathrm{pH} 3.0)$ after each measurement.

\section{CdSe/ZnS QD-conjugated FMDV type $\mathrm{O}$ sdAbs and imaging FMDV in BHK-21 cells}

ZnS-capped CdSe ODs (QDs, $605 \mathrm{~nm}$ ) were synthesized by Wuhan Jiayuan Quantum Dots Co. Ltd. (Wuhan, China). The purified sdAbs were conjugated with QDs in accordance with previously reported procedures [18] to construct two sdAb-QD probes, which were named sdAbc1-QDs and sdAb-c2-QDs. Alexa Flour 488 (AF488) was used to construct sdAb-AF488 probes (sdAb-c1-AF488 
and sdAb-c2-AF488). The conjugation results were determined and visualized using the optimized wide-ranging criteria and agarose gel. For confocal microscopy, a monolayer of baby hamster kidney-21 (BHK-21) cells was cultured in six-well plates and infected with FMDV type O $\left(\right.$ TCID50 $\left.=10^{-6}\right)$. After $1.5 \mathrm{~h}$ of adsorption, the supernatant was discarded, and fresh Dulbecco's modified Eagle's medium supplemented with $2 \%$ fetal bovine serum was added into the wells. The cells were incubated at $37{ }^{\circ} \mathrm{C}$ with $5 \% \mathrm{CO}_{2}$. At $4 \mathrm{~h}$ post infection (h.p.i.), the cells on coverslips were fixed with $4 \%$ paraformaldehyde for $10 \mathrm{~min}$ and then permeabilized with $0.5 \%$ TritonX-100 (Sigma, USA) for $20 \mathrm{~min}$ at $37{ }^{\circ} \mathrm{C}$. Blocking was performed for $2 \mathrm{~h}$ at $37{ }^{\circ} \mathrm{C}$ using PBS containing $5 \%$ skim milk powder to reduce unspecific binding. The cells were probed with sdAb-c1-QDs and sdAb-c2-QDs (diluted in PBS) for $1 \mathrm{~h}$ at $37^{\circ} \mathrm{C}$. The cells were washed thrice with PBS buffer to terminate the reaction and then processed for the sdAb-AF488 probes to further determine the specificity of the sdAbQD probes against FMDV in the cells. A 4,6-diamidino-2phenylindole solution was used to stain the nuclei. The slides were then treated with glycerol jelly mounting medium to prolong storage time. The images were visualized using a laser scanning confocal microscope and saved for further analysis.

\section{Results}

\section{Construction of sdAb library for FMDV type 0}

After three rounds of amplification, a 450 bp fragment was obtained, cloned into the phagemid vector pHEN2, and then transformed into E. coli TG1 cells. A library named NAL-O was produced. Fifteen randomly selected individual clones were tested for the presence of specific $\mathrm{sdAb}$ fragments through PCR. Results demonstrated that all the clones were positive. Local sequencing indicated that each clone contained different complementary determining regions (CDRs). A capacity of $10^{8}$ was calculated on the basis of the diversity and positive clone number.

NAL-O was subjected to four rounds of panning for the phage particles against FMDV type O. Phage recovery increased by more than 100 -fold after the fourth round of panning than after the first one (Table 2), which demonstrated an efficient enriching factor of antigenspecific sdAbs. A total of 131 individual E. coli clones

Table 2 Enriching factor after each round of panning

\begin{tabular}{lccc}
\hline Round & Input $\left({ }^{\mathrm{b}} \mathrm{pfu}\right)$ & Output (pfu) & ${ }^{\text {a }}$ Enriching factor (\%) \\
\hline $1^{\text {st }}$ & $1.0 \times 10^{11}$ & $1.2 \times 10^{4}$ & $1.2 \times 10^{-7}$ \\
$2^{\text {nd }}$ & $9.0 \times 10^{10}$ & $3.2 \times 10^{4}$ & $3.6 \times 10^{-7}$ \\
$3^{\text {rd }}$ & $8.7 \times 10^{10}$ & $2.0 \times 10^{5}$ & $2.3 \times 10^{-6}$ \\
$4^{\text {th }}$ & $2.7 \times 10^{10}$ & $4.3 \times 10^{6}$ & $1.6 \times 10^{-4}$ \\
\hline
\end{tabular}

${ }^{a}$ Enriching factors: Enriching factors = output/input. ${ }^{\mathrm{b}} \mathrm{pfu}$ : plaque-forming unit were randomly chosen from NAL-O and analyzed with monoclonal phage ELISA. Twelve clones yielded high absorbance at $450 \mathrm{~nm}$ (Fig. 2), and local sequencing of the 12 clones helped identify two sdAb gene sequences, which were named sdAb-c1 and sdAb-c2.

The amino acid sequences were subjected to multiple sequence alignment (Fig. 3). Five anti-FMDV Asia 1 sdAbs [GenBank: KC816013, KC816014, KC816015, KC816016, and KC816017] were included in this alignment as references. The alignment results revealed considerable conservation in the framework regions (FR1, FR2, and FR3). The FR2 region in sdAb-c2 contained four hallmark residues (i.e., Phe37, Glu44, Arg45, and Gly47). This indicates that it is derived from a heavy chain antibody [28]. However, sdAb-c1 did not contain these four hallmark residues and was five amino acids shorter than the other sdAbs in the CDR3 region. These findings suggest that sdAb-c1 is a VH-type sdAb.

\section{Expression and purification of sdAb proteins}

The sdAb genes were induced and analyzed using SDSPAGE (Fig. 4a). The sdAbs had an expected molecular weight of approximately $35 \mathrm{kDa}$. The purified sdAb recombinant protein was analyzed with a SDS-PAGE gel (Fig. 4b). Only minor nonspecific contaminations were detected in the eluted fraction. The sdAbs were confirmed with Western blot, and the target protein bands (approximately $35 \mathrm{kDa}$ ) were observed on the PVDF membrane (Fig. 5).

\section{Identification of FMDV type $\mathrm{O}$ sdAbs}

The results of sandwich ELISA showed that the two sdAbs displayed high affinities to FMDV type $\mathrm{O}$ and exhibited no cross-reactivity with serotypes Asia 1 and A in ELISA

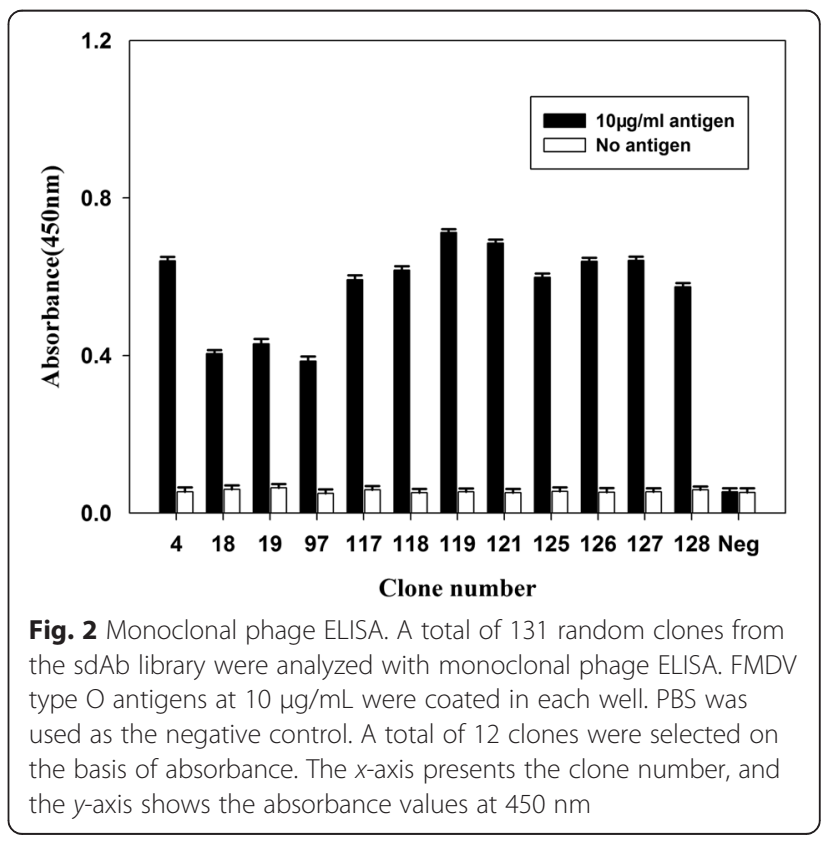




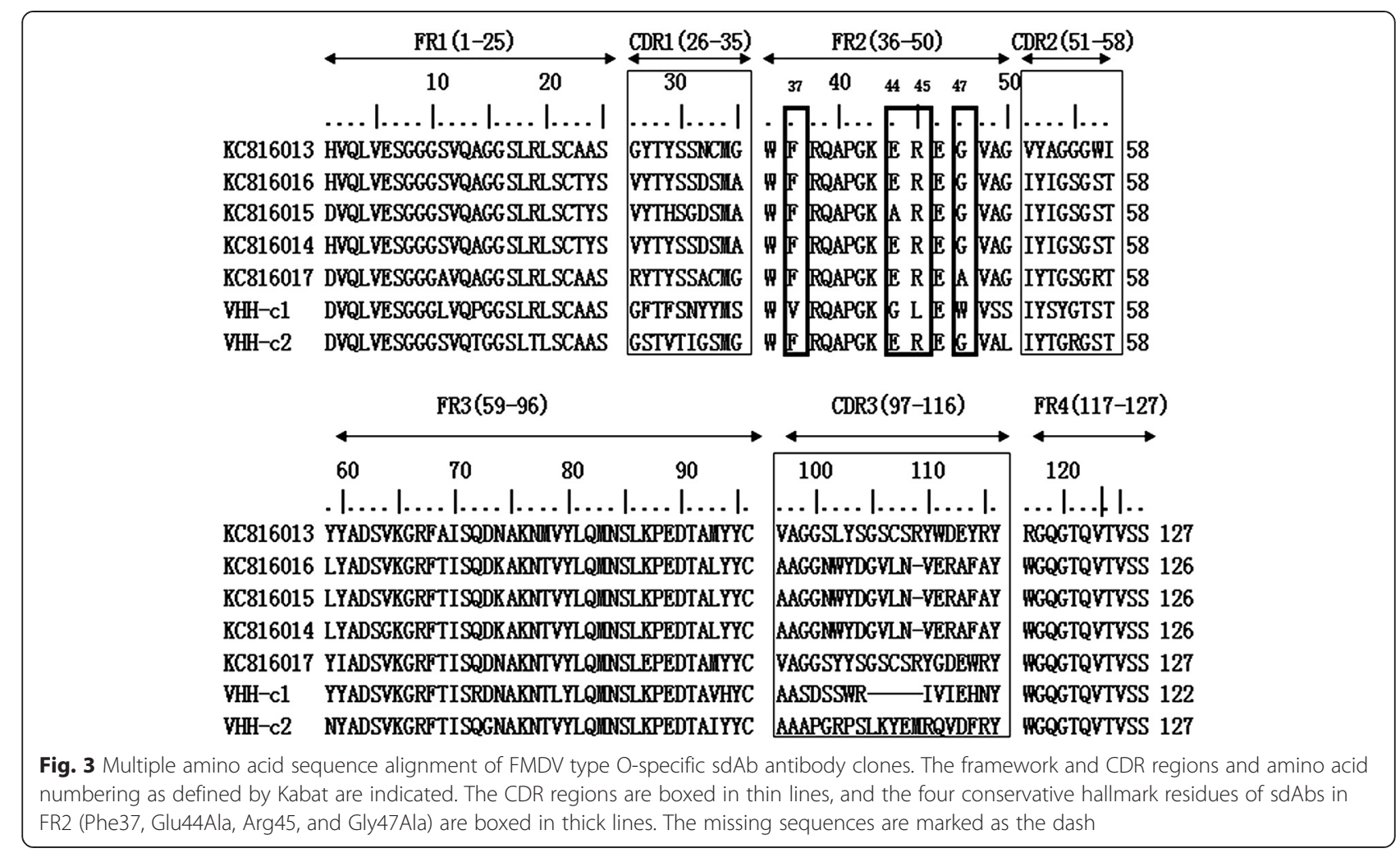

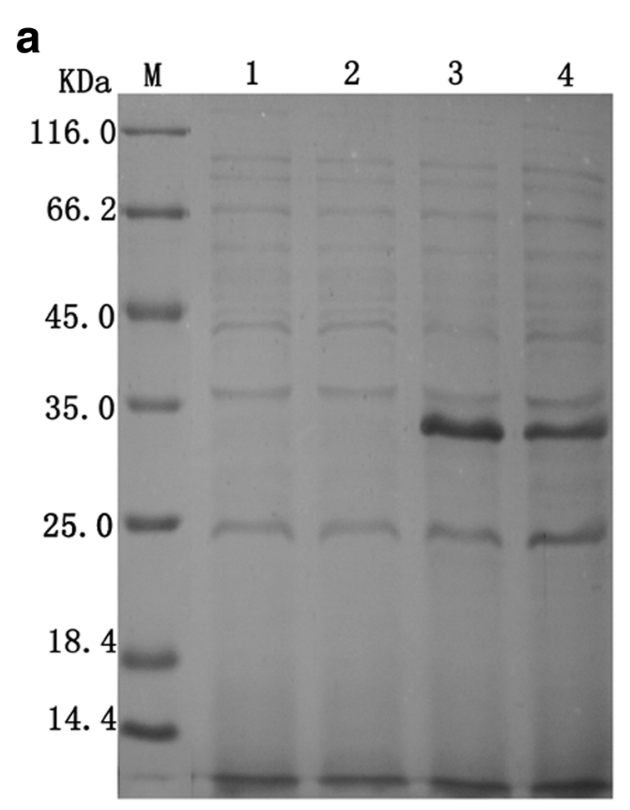

\section{b}

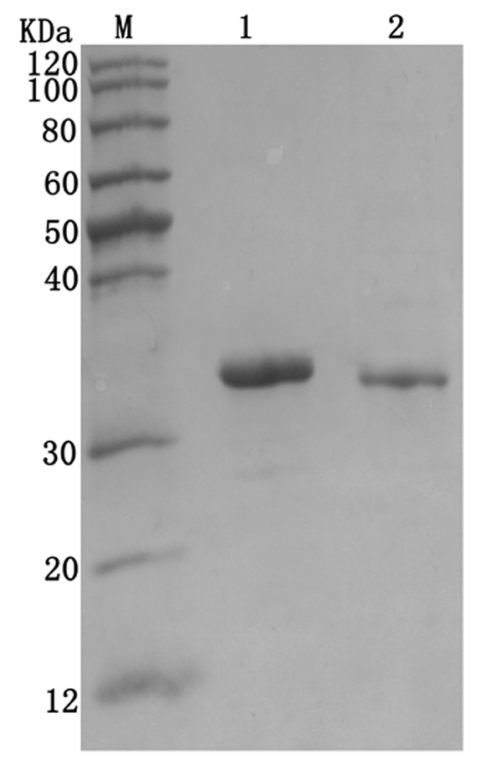

Fig. 4 SDS-PAGE analysis of the expression and purification of sdAbs. a Expression of sdAbs in E. coli. Lanes 1 and 2, bacterial lysates from the $s d A b-c 1$ and sdAb-c2 groups under non-inducing conditions. Lanes 3 and 4, bacterial lysates from the sdAb-c1 and sdAb-c2 groups under inducing conditions. (b) Purification of recombinant sdAbs protein. Lanes 1 and 2, purified sdAb-c1 and sdAb-c2. $M=$ molecular weight markers, size indicated in kilodalton $(\mathrm{kDa})$ 


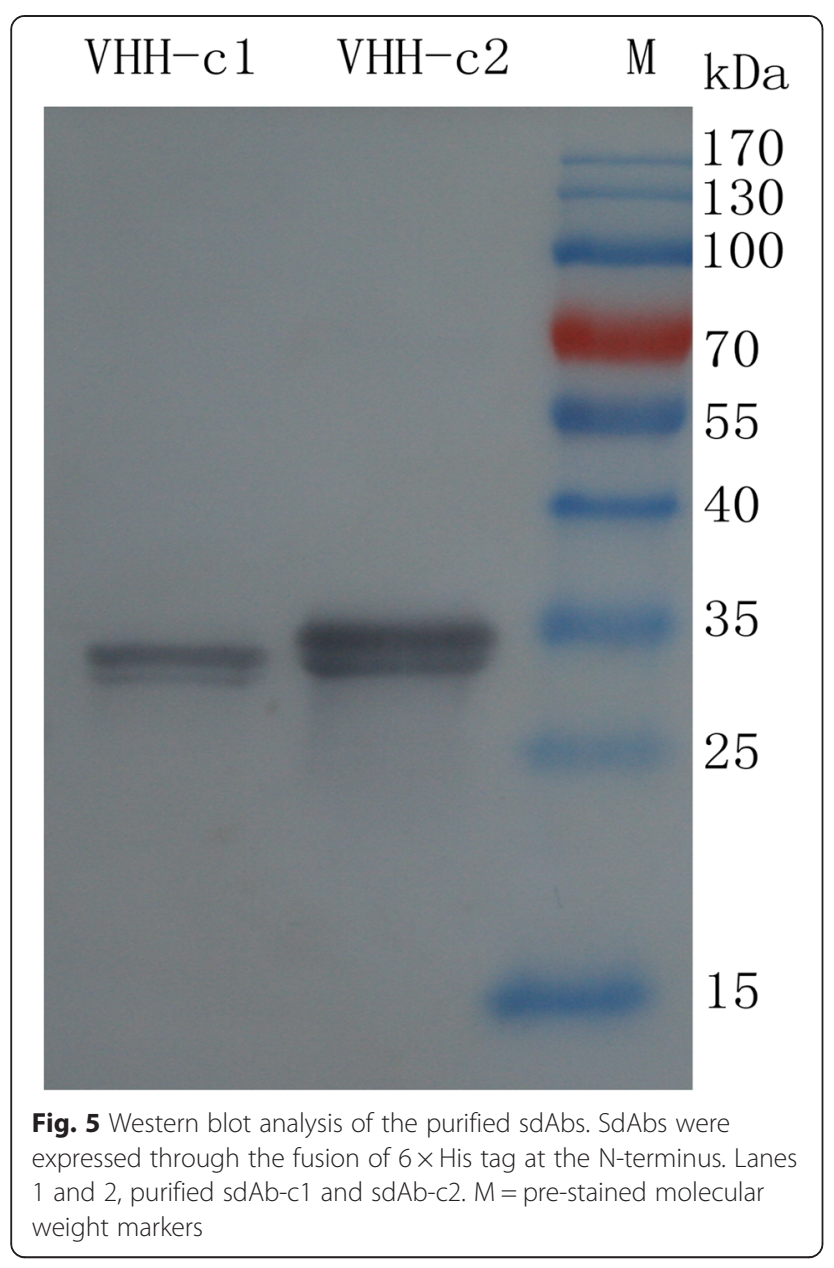

\section{Discussion}

Our group previously screened five sdAbs against FMDV type Asia 1 using the immunized library from C. bactrianus
(Fig. 6). The specificities and high affinities of sdAbs to FMDV serotype $O$ indicated that they could be effective antibodies to this target FMDV serotype.

The association and dissociation rate constants curve were generated by biacore evaluation 4.1 software (additional file 1). The SPR results (Table 3) showed that the affinities of sdAbs ranged from $6.23 \mathrm{nM}$ to $8.24 \mathrm{nM}$, which was within the normal KD range of most sdAbs for their target antigens. These data suggest high affinities to the type $\mathrm{O}$ antigen and are consistent with the results of the monoclonal phage ELISA (Fig. 2).

\section{Imaging FMDV in BHK-21 cells with sdAb-QD probes}

The conjugated products run relatively slow when tested with $1 \%$ agarose gel because of their larger molecular weight than QDs alone (data not shown), indicating that the sdAbs were successfully labeled with QDs. The BHK-21 cells were fixed at 4 h.p.i. and monitored for the presence of FMDV virions using an immunofluorescence assay (IFA). As shown in Fig. 7, the FMDV virions can be observed with sdAb-QD probe staining. To identify the specificity of the sdAb-QD probes in vitro, probes sdAb-QDs and sdAb-AF488 were used to stain the fixed cells in a single trial. The merged images in Fig. 7 indicate that FMDV virions were located at the same area as the sdAb-QDs and sdAb-AF488 probes in BHK-21 cells. Most of the virions were located at one side of the nuclei, which indicated a novel invasion mechanism of FMDV when infecting BHK-21 cells. These results suggest that the sdAbQDs probes are excellent candidates for further research.

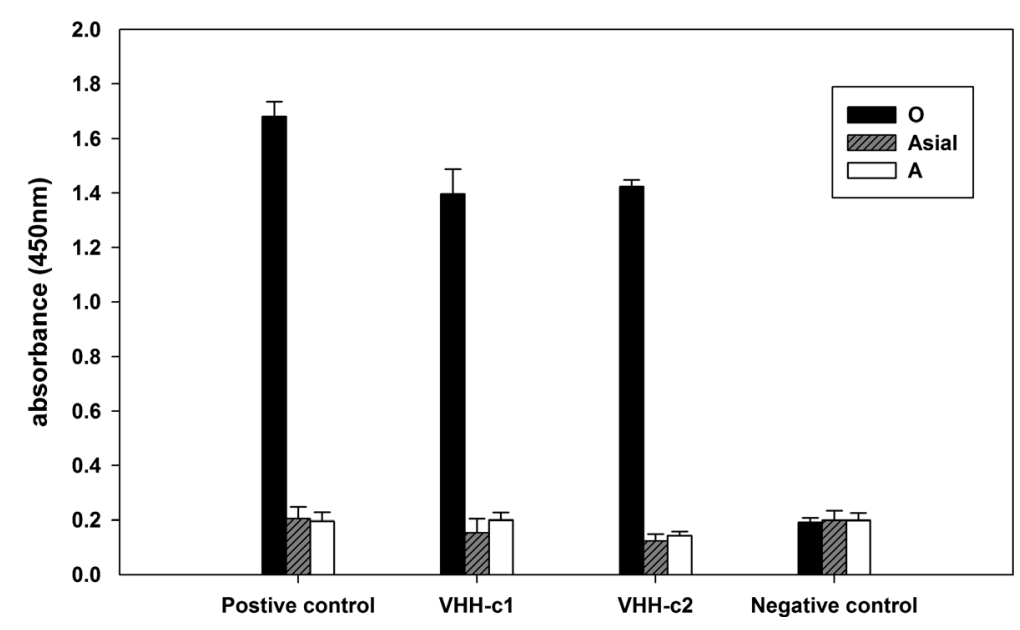

Fig. 6 Serotype specificity identification of sdAbs against FMDV serotype O, Asia 1, and A antigens. All samples were tested for absorbance at $450 \mathrm{~nm}$. The negative camel serum was coated as the negative control, and the polyclonal antibodies against FMDV type O were coated as the positive control. Error bars represent the \pm standard deviation of three independent measurements 
Table 3 Kinetics of sdAbs interaction with FMDV antigens

\begin{tabular}{llll}
\hline $\mathrm{VHH}$ & ${ }^{\mathrm{a}} \mathrm{ka}\left(\mathrm{M}^{-1} \mathrm{~S}^{-1}\right)$ & ${ }^{\mathrm{b}} \mathrm{kd}\left(\mathrm{S}^{-1}\right)$ & ${ }^{{ }^{\mathrm{K} D}}(\mathrm{M})$ \\
\hline $\mathrm{A} 01$ & $1.21 \times 10^{5}$ & $9.99 \times 10^{-4}$ & $8.24 \times 10^{-9}$ \\
D01 & $1.57 \times 10^{5}$ & $9.75 \times 10^{-4}$ & $6.23 \times 10^{-9}$
\end{tabular}

${ }^{\mathrm{a}} \mathrm{ka}$ : association constant. ${ }^{\mathrm{b}} \mathrm{kd}$ : dissociation constant

${ }^{C} \mathrm{KD}$ : equilibrium dissociation constant, $\mathrm{KD}(\mathrm{M})=\mathrm{kd}\left(\mathrm{S}^{-1}\right) / \mathrm{ka}\left(\mathrm{M}^{-1} \mathrm{~S}^{-1}\right)$

with Asia 1 virus-like particles. Three of the sdAbs showed high binding affinities and high solubilities. They proved powerful when detecting the virus in host cells. However, FMDV is divided into seven immunologically distinct serotypes that do not share cross-immunity. Serotype $O$ is the most globally prevalent of the seven serotypes of FMDV. Several outbreaks of serotype $O$ have recently occurred in China [29] and neighboring countries [30], suggesting the urgent need to prevent this FMDV serotype. In this work, we constructed a sdAb library for FMDV serotype $O$ and selected the special sdAbs against this serotype. The special sdAbs from this library may serve an important function in the research of serotype O FMDV infection. Antibodies are valuable tools for visualizing antigens in cells because of their high affinity and high specificity [31, 32]. Conventional antibodies and some of their derivatives have been tested as targeting agents, but their development are hindered by disadvantages such as poor stability, aggregation, and high production cost [17]. The use of sdAbs can overcome these disadvantages and enable new functional studies. SdAbs are the smallest functional antibodies and are easily produced in prokaryotic and eukaryotic microorganisms [8, 10, 33].
In addition, they can be widely used in immunofluorescence techniques to capture antigens because of their high affinity [34, 35]. The CDR3 of sdAbs is longer than that of conventional antibodies. Thus, this CDR3 forms a large loop that may help the sdAbs extend in cavities on antigens to bind epitopes inaccessible to conventional antibodies. The sdAbs are also particularly suited for intrabody production because they fold efficiently into functional antigenbinding entities even inside the reducing environment of the cytoplasm [36]. As previously reported [35], sdAbs were fused with fluorescent proteins to generate nanoantibodies that could be expressed in living cells and used to target and trace antigens in live cells. To date, antigens such as protein toxins and poisons, small molecule toxins, and other haptens, viruses, pathogenic bacteria, and parasites are reportedly targeted by sdAbs for diagnosis and therapy [37].

SdAbs can be screened from both immune libraries and non-immune libraries. However, sdAbs derived from nonimmune libraries show low affinities for their target antigens [7, 38, 39]. Most immune sdAb libraries are from camels because of their manageability. In this work, two camels devoid of any FMDV vaccine immunization were chosen from the Gobi Desert in Western China. A previous report [40] explained that dromedary camels have low susceptibility to FMDV serotype $\mathrm{O}$ and do not transmit FMDV to the susceptible species even through close contact. In addition, high titers of antibodies after the immunization with FMDV antigens make Bactrian

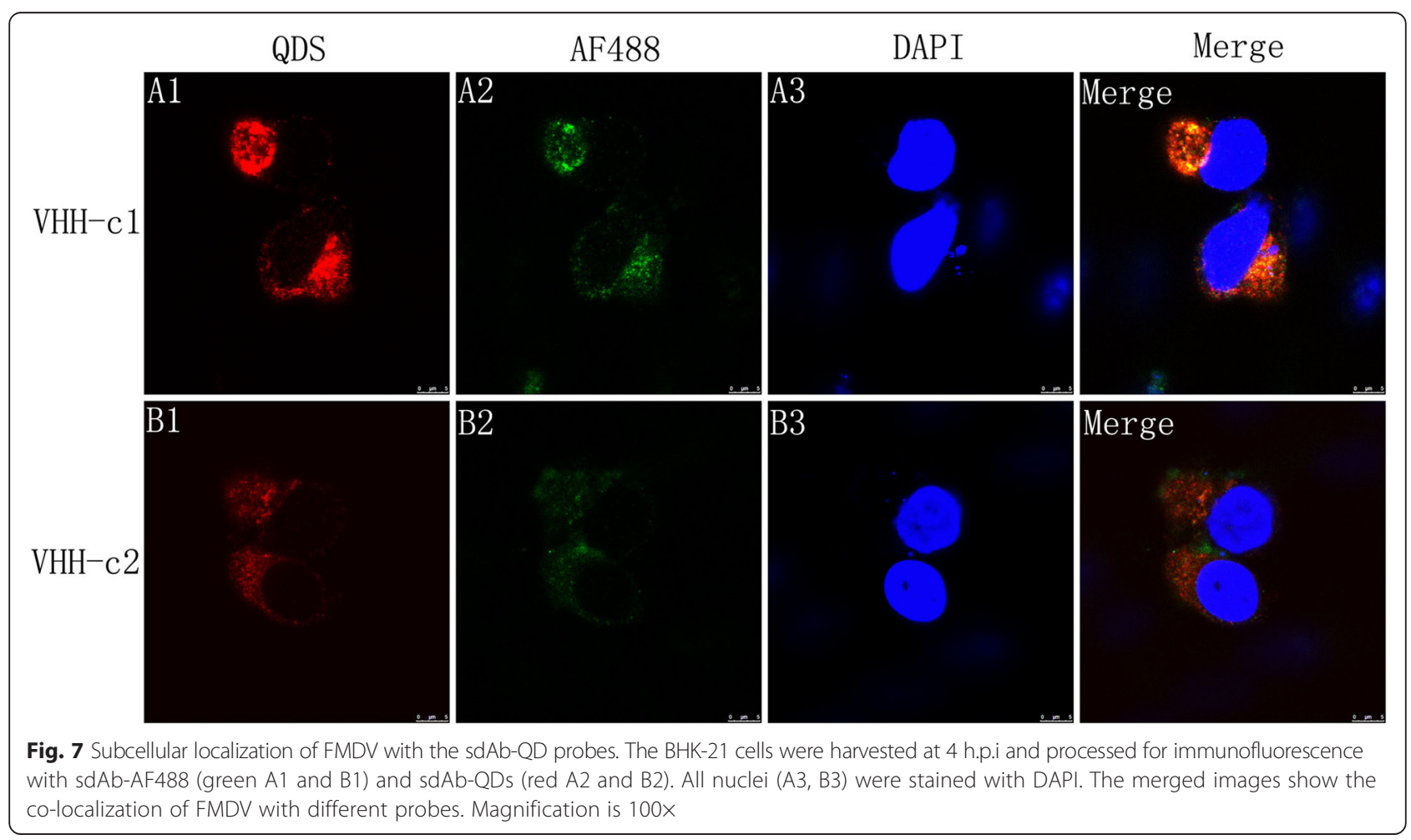


camels ideal candidate animals for the production of sdAbs against FMDV. The camels were injected with FMDV type $O$ vaccine to yield an immune library against FMDV. Two unique sdAbs with high affinities to type O FMDV were selected and tested as an efficient tool for targeting specific antigens through phage display technology.

QDs are ideal inorganic luminescent materials that have been widely used for in vivo molecular and cellular imaging [41]. In the present work, special sdAbs were conjugated with QDs to generate useful probes for targeting and imaging the FMDV type $\mathrm{O}$ virions while infecting BHK-21 cells. BHK-21 cells were fixed at 4 h.p.i., and the FMDV virions were monitored with special probes and a laser scanning confocal microscope. As shown in the images, most of the viruses were clustered at one side of the nuclei. This result is consistent with previous reports [42]. Host cell membranes are rearranged to create vesicular structures when FMDV infects the host cells. Virus genome replication occurs on the vesicular structures. Upon FMDV infection of the cell, cytoplasmic components such as free ribosomes, fragmented rough endoplasmic reticulum, Golgi and smooth membrane-bound vesicles accumulated on one side of the nucleus. The collapse of cellular organelles to one side of the cell was considered as the preparation for virus replication.

\section{Conclusions}

We successfully constructed a specific anti-FMDV type O sdAb immune library using the phage display technology. Two sdAbs from this library were identified through Western blot, ELISA, and SPR. Meanwhile, the two sdAbs were conjugated with QDs to form QD-sdAb probes. The results of the IFA demonstrate that the sdAbs are candidate probes for tracing FMDV type O virions in BHK-21 cells. This study expanded the application of sdAbs as in vivo tools for virus infection and replication.

\section{Additional file}

Additional file 1: Figure S1. Binding kinetic measurement. Samples were serial diluted in different concentrations $(25,50,75,100$ and $150 \mathrm{nM})$. Association and dissociation constants of $\mathrm{VHH}$ s were determined separately through SPR measurement using Biacore 3000. The curve shows the result of $\mathrm{VHH}-\mathrm{C} 2$.

\section{Abbreviations}

FMD: Foot-and-mouth disease; FMDV: Foot-and-mouth disease virus; QD: Quantum dot; sdAb: Single-domain antibody; $V H H$ : The variable domain of the heavy chain from the heavy-chain antibodies; ELISA: Enzyme-linked immunosorbent assay; SPR: Surface plasmon resonance assay; BHK: Baby hamster kidney.

\section{Competing interests}

The authors declare that they have no competing interests.

\section{Authors' contributions}

$X T L, S H Y$, and DW participated in the design of the study and drafted the manuscript. SLY conducted the surface plasmon resonance assay. PD, JHG, and JJH contributed reagents, materials, and analysis tools. YJS and JPC participated in the data analysis. All authors read and approved the final manuscript.

\section{Acknowledgements}

This work was supported by the China Agriculture Research System (CARS-39) and Basic Research Project (No. 2013FY113300).

Received: 13 October 2014 Accepted: 12 May 2015

Published online: 22 May 2015

\section{References}

1. Yang PC, Chu RM, Chung WB, Sung HT. Epidemiological characteristics and financial costs of the 1997 foot-and-mouth disease epidemic in Taiwan. Vet Rec. 1999;145:731-4.

2. Thompson D, Muriel P, Russell D, Osborne P, Bromley A, Rowland M, et al. Economic costs of the foot and mouth disease outbreak in the United Kingdom in 2001. Rev Sci Tech. 2002;21:675-87.

3. Garland A, Donaldson A. Foot-and-mouth disease. Surveillance. 1990;17:6-8.

4. Bachrach HL. Foot-and-mouth disease. Ann Rev Microbiol. 1968;22:201-44.

5. Hamers-Casterman C, Atarhouch T, Muyldermans S, Robinson G, Hamers C, Songa EB, et al. Naturally occurring antibodies devoid of light chains. Nature. 1993;363:446-8.

6. Greenberg AS, Avila D, Hughes M, Hughes A, McKinney EC, Flajnik MF. A new antigen receptor gene family that undergoes rearrangement and extensive somatic diversification in sharks. Nature. 1995;374:168-73.

7. Harmsen MM, De Haard HJ. Properties, production, and applications of camelid single-domain antibody fragments. Appl Microbiol Biotechnol. 2007;77:13-22.

8. Rahbarizadeh F, Rasaee MJ, Forouzandeh-Moghadam M, Allameh AA. High expression and purification of the recombinant camelid anti-MUC1 single domain antibodies in Escherichia coli. Protein Expr Purif. 2005;44:32-8.

9. Frenken LG, van der Linden RH, Hermans PW, Bos JW, Ruuls RC, de Geus B, et al. Isolation of antigen specific llama $\mathrm{VHH}$ antibody fragments and their high level secretion by Saccharomyces cerevisiae. J Biotechnol. 2000;78:11-21.

10. Ismaili A, Jalali-Javaran M, Rasaee MJ, Rahbarizadeh F, Forouzandeh-Moghadam M, Memari HR. Production and characterization of anti-(mucin MUC1) single-domain antibody in tobacco (Nicotiana tabacum cultivar Xanthi). Biotechnol Appl Biochem. 2007;47:11-9.

11. Revets H, De Baetselier P, Muyldermans S. Nanobodies as novel agents for cancer therapy. Expert Opinion Biol Ther. 2005;5:111-24.

12. Cortez-Retamozo V, Lauwereys M, Hassanzadeh Gh G, Gobert M, Conrath K, Muyldermans $\mathrm{S}$, et al. Efficient tumor targeting by single-domain antibody fragments of camels. Int J Cancer. 2002;98:456-62.

13. Leutwyler WK, Bürgi SL, Burgl H. Semiconductor clusters, nanocrystals, and quantum dots. Science. 1996;271:933-7.

14. Voura EB, Jaiswal JK, Mattoussi H, Simon SM. Tracking metastatic tumor cell extravasation with quantum dot nanocrystals and fluorescence emission-scanning microscopy. Nat Med. 2004;10:993-8.

15. Lidke DS, Nagy P, Heintzmann R, Arndt-Jovin DJ, Post JN, Grecco HE, et al. Quantum dot ligands provide new insights into erbB/HER receptor-mediated signal transduction. Nature Biotechnol. 2004;22:198-203.

16. Jaiswal JK, Mattoussi H, Mauro JM, Simon SM. Long-term multiple color imaging of live cells using quantum dot bioconjugates. Nature Biotechnol. 2002;21:47-51.

17. Zaman MB, Baral TN, Zhang J, Whitfield D, Yu K. Single-domain antibody functionalized CdSe/ZnS quantum dots for cellular imaging of cancer cells. J Physical Chem C. 2008;113:496-9.

18. Yin S, Yang S, Shang Y, Sun S, Zhou G, Jin Y, et al. Characterization of Asia $1 \mathrm{sdAb}$ from Camels Bactrianus (C. bactrianus) and Conjugation with Quantum Dots for Imaging FMDV in BHK-21 Cells. PloS One. 2013;8, e63500.

19. English D, Andersen BR. Single-step separation of red blood cells. Granulocytes and mononuclear leukocytes on discontinuous density gradients of Ficoll-Hypaque. J Immunol Methods. 1974;5:249-52.

20. Hoogenboom HR, Griffiths AD, Johnson KS, Chiswell DJ, Hudson P, Winter G. Multi-subunit proteins on the surface of filamentous phage: methodologies for displaying antibody (Fab) heavy and light chains. Nucleic Acids Res. 1991;19:4133-7. 
21. Hayhurst A, Happe S, Mabry R, Koch Z, Iverson BL, Georgiou G. Isolation and expression of recombinant antibody fragments to the biological warfare pathogen Brucella melitensis. J Immunol Methods. 2003;276:185-96.

22. Goffinet M, Chinestra P, Lajoie-Mazenc I, Medale-Giamarchi C, Favre G, Faye JC. Identification of a GTP-bound Rho specific SCFv molecular sensor by phage display selection. BMC Biotechnol. 2008:8:34

23. Hoogenboom HR, de Bruine AP, Hufton SE, Hoet RM, Arends JW, Roovers RC. Antibody phage display technology and its applications. Immunotechnology. 1998:4:1-20.

24. Yang S, Shang Y, Yin S, Tian H, Chen Y, Sun S, et al. Selection and identification of single-domain antibody fragment against capsid protein of porcine circovirus type 2 (PCV2) from C. bactrianus. Vet Immunol Immunopathol. 2014;160:12-9.

25. Yin S, Sun S, Yang S, Shang Y, Cai X, Liu X. Self-assembly of virus-like particles of porcine circovirus type 2 capsid protein expressed from Escherichia coli. Virol J. 2010;7:166.

26. Butt TR, Edavettal SC, Hall JP, Mattern MR. SUMO fusion technology for difficult-to-express proteins. Protein Expr Purif. 2005;43:1-9.

27. Liu JL, Hu ZQ, Xing S, Xue S, Li HP, Zhang JB, et al. Attainment of 15-fold higher affinity of a Fusarium-specific single-chain antibody by directed molecular evolution coupled to phage display. Mol Biotechnol. 2012;52:111-22.

28. Vu KB, Ghahroudi MA, Wyns L, Muyldermans S. Comparison of Ilama VH sequences from conventional and heavy chain antibodies. Mol Immunol. 1997;34:1121-31

29. Zheng H, He J, Guo J, Jin Y, Yang F, Lv L, et al. Genetic characterization of a new pandemic Southeast Asia topotype strain of serotype $O$ foot-and-mouth disease virus isolated in China during 2010. Virus Genes. 2012;44:80-8.

30. Valdazo-Gonzalez B, Timina A, Scherbakov A, Abdul-Hamid NF, Knowles NJ, King DP. Multiple introductions of serotype $O$ foot-and-mouth disease viruses into East Asia in 2010-2011. Vet Res. 2013:44:76.

31. Bignami A, Eng LF, Dahl D, Uyeda CT. Localization of the glial fibrillary acidic protein in astrocytes by immunofluorescence. Brain Res. 1972;43:429-35.

32. Henle G, Henle W. Immunofluorescence in cells derived from Burkitt's lymphoma. J Bacteriol. 1966;91:1248-56.

33. Rahbarizadeh F, Rasaee MJ, Forouzandeh M, Allameh AA. Over expression of anti-MUC1 single-domain antibody fragments in the yeast Pichia pastoris. Mol Immunol. 2006;43:426-35.

34. Olichon A, Surrey T. Selection of genetically encoded fluorescent single domain antibodies engineered for efficient expression in Escherichia coli. J Biol Chem. 2007;282:36314-20.

35. Rothbauer U, Zolghadr K, Tillib S, Nowak D, Schermelleh L, Gahl A, et al. Targeting and tracing antigens in live cells with fluorescent nanobodies. Nat Methods. 2006;3:887-9.

36. Gueorguieva D, Li S, Walsh N, Mukerji A, Tanha J, Pandey S. Identification of single-domain, Bax-specific intrabodies that confer resistance to mammalian cells against oxidative-stress-induced apoptosis. FASEB J. 2006:20:2636-8.

37. Wesolowski J, Alzogaray V, Reyelt J, Unger M, Juarez K, Urrutia M, et al. Single domain antibodies: promising experimental and therapeutic tools in infection and immunity. Med Microbiol Immunol. 2009;198:157-74.

38. Pini A, Bracci L. Phage display of antibody fragments. Curr Protein Pept Sci. 2000;1:155-69.

39. Hoogenboom HR. Selecting and screening recombinant antibody libraries. Nat Biotechnol. 2005;23:1105-16.

40. Alexandersen S, Wernery U, Nagy P, Frederiksen T, Normann P. Dromedaries (Camelus dromedarius) are of low susceptibility to inoculation with foot-and-mouth disease virus serotype O. J Comp Pathol. 2008;139:187-93.

41. Huo Q. A perspective on bioconjugated nanoparticles and quantum dots. Colloids Surf B Biointerfaces. 2007:59:1-10.

42. Monaghan $\mathrm{P}$, Cook $\mathrm{H}$, Jackson $\mathrm{T}$, Ryan M, Wileman $\mathrm{T}$. The ultrastructure of the developing replication site in foot-and-mouth disease virus-infected BHK-38 cells. J Gen Virol. 2004;85:933-46.

\section{Submit your next manuscript to BioMed Central and take full advantage of:}

- Convenient online submission

- Thorough peer review

- No space constraints or color figure charges

- Immediate publication on acceptance

- Inclusion in PubMed, CAS, Scopus and Google Scholar

- Research which is freely available for redistribution

Submit your manuscript at www.biomedcentral.com/submit 